\title{
Inhaled iloprost suppresses the cardinal features of asthma via inhibition of airway dendritic cell function
}

\author{
Marco Idzko,, ${ }^{1,2}$ Hamida Hammad, ${ }^{1}$ Menno van Nimwegen, ${ }^{1}$ Mirjam Kool, ${ }^{1}$ \\ Nanda Vos, ${ }^{1}$ Henk C. Hoogsteden, ${ }^{1}$ and Bart N. Lambrecht ${ }^{1}$ \\ ${ }^{1}$ Department of Pulmonary Medicine, Erasmus University Medical Center, Rotterdam, The Netherlands. \\ 2Department of Pneumology, University of Freiburg, Freiburg, Germany.
}

\begin{abstract}
Inhalation of iloprost, a stable prostacyclin $\left(\mathrm{PGI}_{2}\right)$ analog, is a well-accepted and safe treatment for pulmonary arterial hypertension. Although iloprost mainly acts as a vasodilator by binding to the I prostanoid (IP) receptor, recent evidence suggests that signaling via this receptor also has antiinflammatory effects through unclear mechanisms. Here we show in a murine model of asthma that iloprost inhalation suppressed the cardinal features of asthma when given during the priming or challenge phase. As a mechanism of action, iloprost interfered with the function of lung myeloid DCs, critical antigen-presenting cells of the airways. Iloprost treatment inhibited the maturation and migration of lung DCs to the mediastinal LNs, thereby abolishing the induction of an allergen-specific Th2 response in these nodes. The effect of iloprost was DC autonomous, as iloprost-treated DCs no longer induced Th2 differentiation from naive $T$ cells or boosted effector cytokine production in primed Th2 cells. These data should pave the way for a clinical effectiveness study using inhaled iloprost for the treatment of asthma.
\end{abstract}

\section{Introduction}

Asthma is a Th2 lymphocyte-mediated inflammatory airway disease characterized by airway eosinophilia, increased mucus production by goblet cells, and structural remodeling of the airway wall. This leads to variable airway obstruction and bronchial hyperresponsiveness (BHR) to nonspecific stimuli. In the airways of allergen-challenged asthmatics, there is increased production of PGs (1-3). PGs are locally acting autacoids generated by stepwise conversion of arachidonic acid into 2 short-lived intermediates, prostaglandin $\mathrm{G}$ and prostaglandin $\mathrm{H}$, through the action of the COX enzymes COX-1 and COX-2 $(1,4,5)$. These intermediate products can be further metabolized by specific enzymes to a series of products including $\mathrm{PGD}_{2}, \mathrm{PGE}_{2}, \mathrm{PGF}_{2 \alpha}$, prostacyclin $\left(\mathrm{PGI}_{2}\right)$, and $\mathrm{TXA}_{2}(6)$. PGs have pleiotropic roles in physiologic and pathophysiologic processes, including inflammation and allergic responses $(4,5)$. In asthma, PGs are generally regarded as proinflammatory molecules, but this view has recently been challenged by work showing that $\mathrm{PGE}_{2}$ can have antiinflammatory and antiasthmatic effects when acting on the E prostanoid receptor 3 (7) as well as by studies showing that COX-1- and COX-2-deficient mice or mice treated with COX inhibitors had exaggerated inflammatory airway responses and BHR in a murine model of asthma (8-10). These data support the concept that endogenous PGs play a regulatory role in the allergic response with an overall balance favoring suppression of the asthmatic response. As inhibition of COX activity leads to a reduction in a wide range of $\mathrm{PGs}$ (e.g., $\mathrm{PGD}_{2}$, $\mathrm{PGE}_{2}$, and $\mathrm{PGI}_{2}$ ) and downstream metabolites (e.g., the cyclopentenone PG 15 -deoxy- $\left.\Delta^{12,14}-\mathrm{PGJ}_{2}\right)$, the exact PG or its specific receptor

Nonstandard abbreviations used: BAL, bronchoalveolar lavage; BHR, bronchial hyperresponsiveness; IP, I prostanoid; i.t., intratracheal(ly); mDC, myeloid DC; MLN mediastinal LN; pDC, plasmacytoid DC; PenH, enhanced pause; $\mathrm{PGI}_{2}$, prostacyclin. Conflict of interest: The authors have declared that no conflict of interest exists. Citation for this article: J. Clin. Invest. 117:464-472 (2007). doi:10.1172/JCI28949. that might be involved in protecting the airways and dampening inflammation during allergen challenge is currently unknown. In this regard, $\mathrm{PGI}_{2}$ may be of great interest because large amounts are produced in a COX-2-dependent manner during IgE-mediated allergic reactions in humans $(11,12)$ and in mice with Th2-mediated airway inflammation (8). In support of an antiinflammatory effect of $\mathrm{PGI}_{2}$, mice deficient in the $\mathrm{PGI}_{2}$-exclusive I prostanoid (IP) receptor have exaggerated features of acute and chronic experimental asthma, including increased $\operatorname{BHR}(13,14)$. Whether $\mathrm{PGI}_{2}$ would act mainly during sensitization to inhaled allergen or during the allergen challenge phase could not be addressed in IP-deficient mice. In all these models, the precise mechanism of action by which $\mathrm{PGI}_{2}$ might suppress airway inflammation has not to our knowledge been elucidated previously.

Here we used inhaled iloprost, a stable $\mathrm{PGI}_{2}$ analog used clinically as a drug treatment for patients with pulmonary arterial hypertension (15), to study the mechanism of action by which IP triggering suppresses asthma features. We focused particularly on DCs, the most powerful antigen-presenting cells of the immune system. Lung DCs have the unique capacity of stimulating both naive $T$ cells during sensitization and primed Th2 cells during recall responses in the airways (16-20). In the induced absence of DCs, all the cardinal features of asthma such as Th2 cytokinedependent airway eosinophilia, goblet cell hyperplasia, and BHR disappear $(21,22)$. Our results show that iloprost suppressed not only the development of asthma when given during the sensitization phase, but also the salient features of experimental asthma during the challenge phase by interfering with the function of these antigen-presenting cells.

\section{Results}

Effect of iloprost inhalation during OVA challenge on airway inflammation and BHR. We investigated whether local application of iloprost (in clinically relevant doses, also used for the treatment of pulmo- 
A

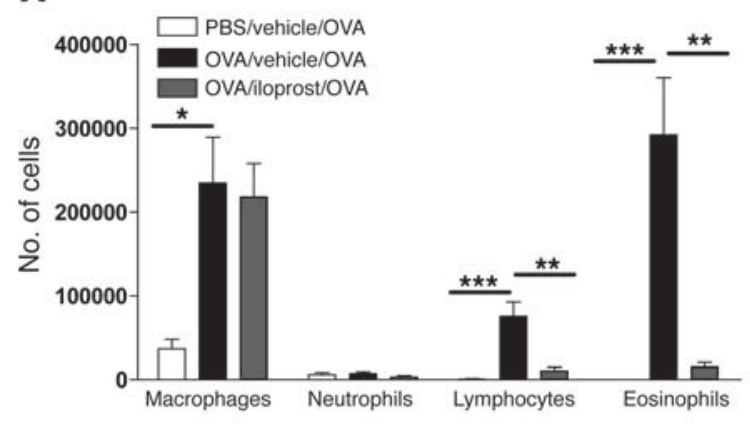

B PBS/vehicle/OVA

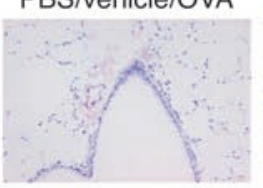

OVA/vehicle/OVA
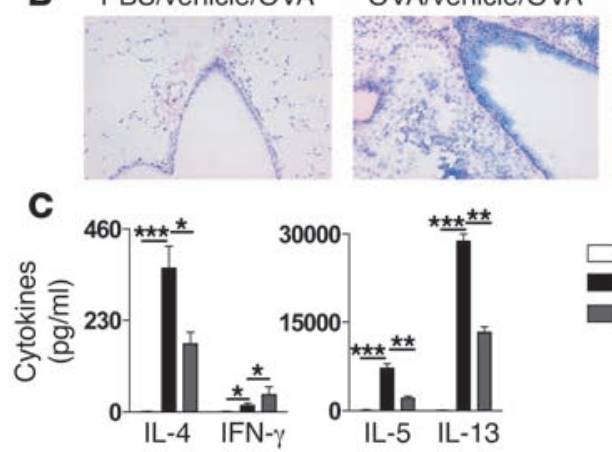

OVA/iloprost/OVA

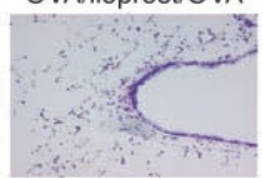

E
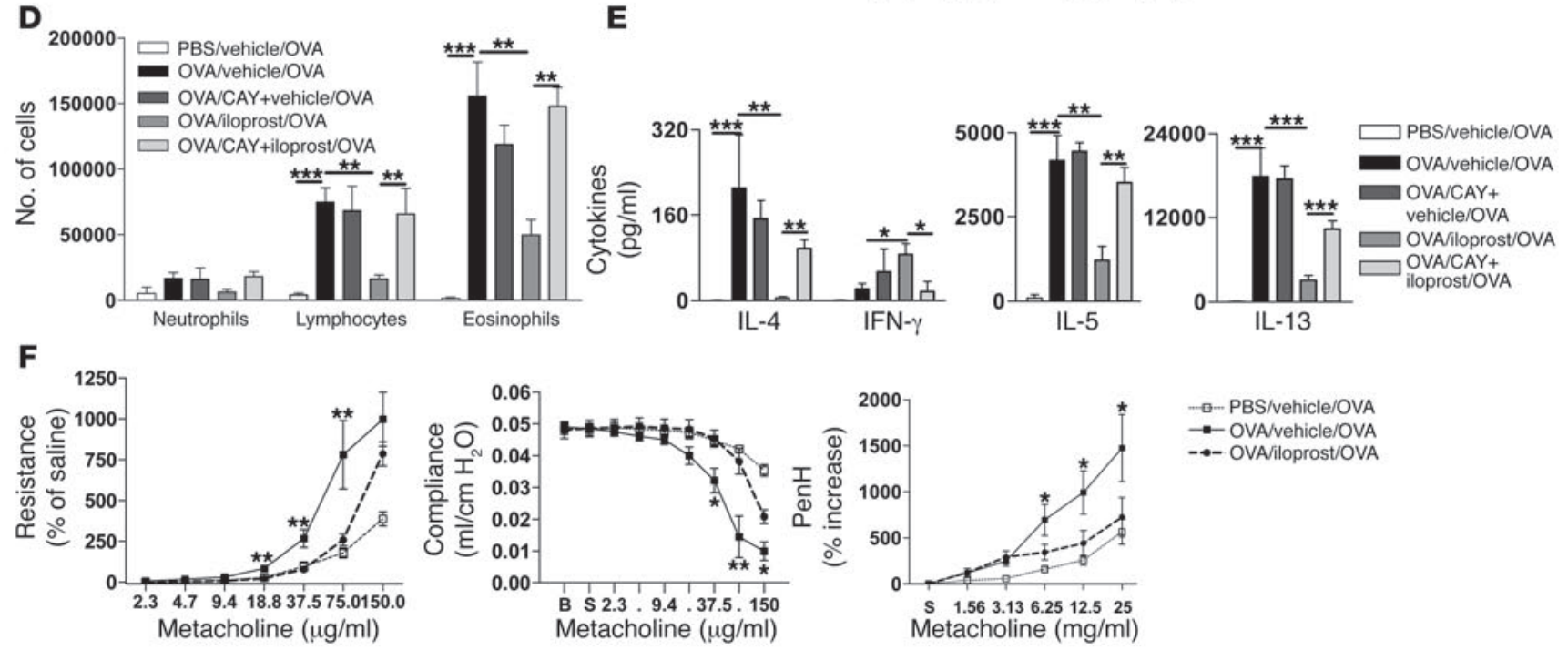

$\rightarrow-$ PBS/vehicle/OVA

- OVA/vehicle/OVA

\section{Figure 1}

Local administration of iloprost suppresses asthma features. Mice were sensitized by i.p. injection of OVA/alum (see Methods) on days 0 and 7 and were exposed on days 19-21 to OVA aerosols. Prior to each aerosol, mice received an i.t. injection of vehicle, CAY10449 plus vehicle (OVA/CAY+vehicle/OVA), $0.2 \mu \mathrm{g}$ iloprost, or CAY10449 plus iloprost. Labels indicate sensitization/treatment/challenge. (A and D) BAL fluid was analyzed by flow cytometry. (B) May-Grunwald-Giemsa staining of lung sections. (C and E) Cytokine production in MLN cells restimulated in vitro for 4 days with OVA. (F) BHR to various doses of i.v. metacholine was assessed for changes in dynamic resistance and lung compliance and $\mathrm{BHR}$ to inhaled metacholine for PenH responses was assessed 24 hours after the last antigen exposure were measured. Data are mean \pm SEM; $n=8$ mice per group. ${ }^{*} P<0.05 ;{ }^{* *} P<0.01 ;{ }^{* *} P<0.001$.

nary hypertension) could influence the development of experimental asthma in already sensitized mice. Sensitization to OVA was induced using i.p. injection of OVA (or sham PBS) in the Th2 adjuvant alum, and mice were subsequently challenged 3 times 10 days later (21). As expected, OVA-sensitized mice treated with vehicle prior to OVA aerosol challenge developed bronchoalveolar lavage (BAL) fluid eosinophilia and lymphocytosis accompanied by enhanced Th2 cytokine production in the mediastinal LNs (MLNs), an effect not seen in sham-sensitized mice. The intratracheal (i.t.) administration of $0.20 \mu \mathrm{g}$ iloprost ( $80 \mu \mathrm{l}$ of a $2.5 \mu \mathrm{g} / \mathrm{ml}$ solution) 30 minutes prior to each allergen challenge resulted in a significant reduction of the lymphocyte and eosinophil infiltrate into the BAL compartment (Figure $1 \mathrm{~A}$ ) and in a reduction of peribronchial and perivascular inflammation and goblet cell hyperplasia on lung sections (Figure 1B). The reduction of airway inflammation in iloprost-treated mice was accompanied by mildly but significantly reduced levels of IL-4, IL-5, and IL-13 in the MLNs and a weak increase in IFN- $\gamma$ production (Figure 1C), while the concentration of IL-10 was not significantly changed (data not shown). The effect of iloprost on all these parameters was dose dependent (see Supplemental Figure 1; supplemental material available online with this article; doi:10.1172/JCI28949DS1).

To prove the involvement of the IP receptor in the iloprostinduced inhibition of asthmatic inflammation, some mice received $200 \mu \mathrm{g}$ of the selective IP receptor antagonist CAY10449 $(10 \mathrm{mg} / \mathrm{kg})$ i.t. prior to iloprost application (23). As shown in Figure 1, D and E, blocking of the IP receptor completely reversed the iloprostinduced suppression of eosinophilic airway inflammation.

BHR to nonspecific stimuli like metacholine is one of the defining symptoms of allergic asthma. As shown in Figure 1F, the allergen challenge of OVA-sensitized mice induced a significant change in responsiveness to i.v. metacholine compared with sham-sensitized mice, as measured 24 hours after the last OVA aerosol challenge by invasive measurement of dynamic resistance and compliance in mechanically ventilated mice. Inhalation of iloprost prior to each allergen challenge markedly attenuated the OVA-induced change in metacholine responsiveness. In OVAsensitized mice, iloprost inhalation prior to each PBS challenge 


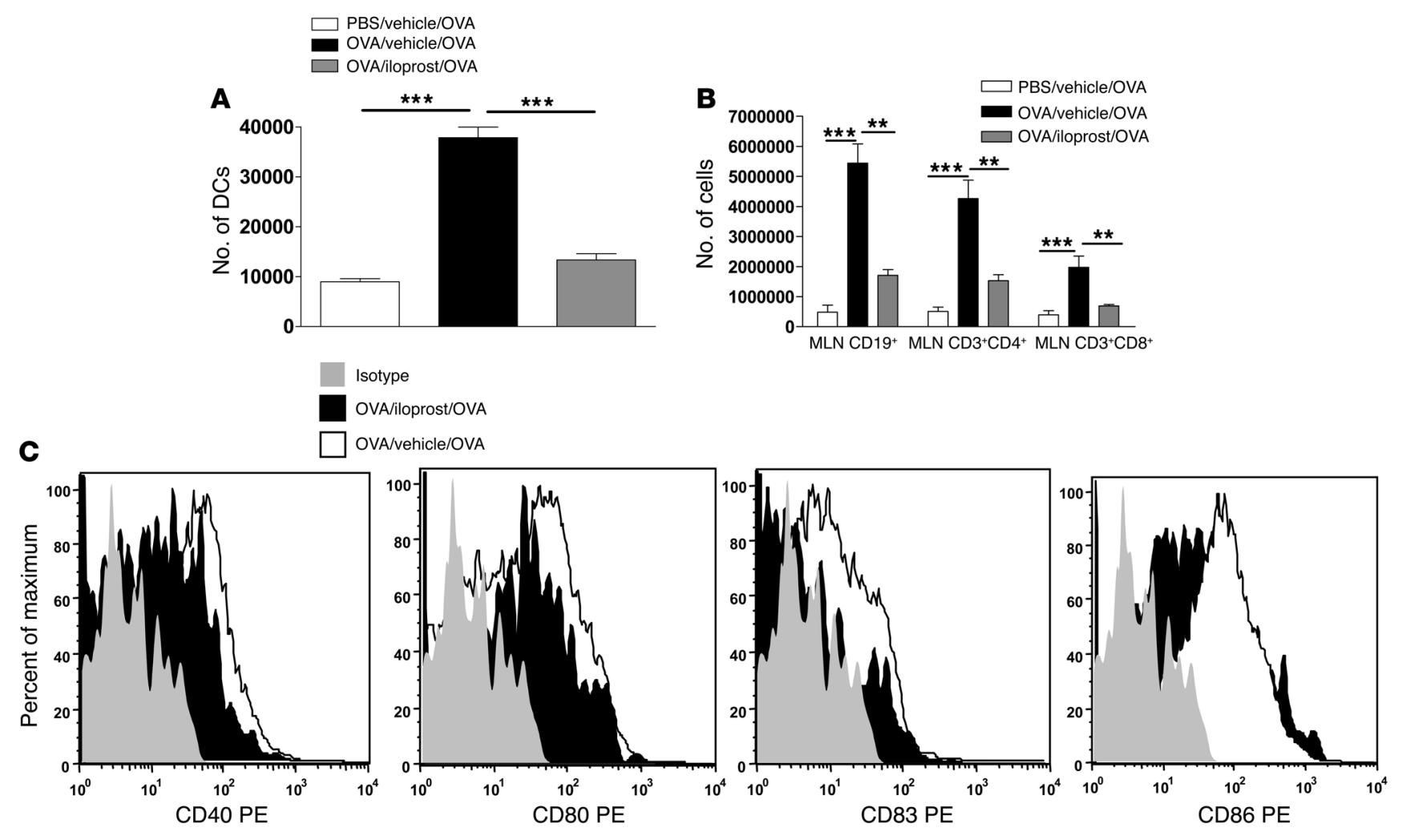

Figure 2

Effect of iloprost treatment on distribution of DCs. Single-cell suspensions from MLNs were stained for DCs (A) or B and T cells (B) and analyzed by flow cytometry. Experiments were set up as in Figure 1. Labels indicate sensitization/treatment/challenge. Data (mean \pm SEM) were calculated as absolute number of cells. ${ }^{* \star} P<0.01 ;{ }^{* \star *} P<0.001$. (C) Iloprost inhibited the maturation of lung DCs in vivo. A single-cell suspension was prepared from the lungs, and CD11 $+\mathrm{MHCl}$ hi lung DCs were analyzed for their expression of CD40, CD80, CD83, and CD86. Data from 1 representative experiment of 3 is shown.

did not significantly modify lung function parameters compared with vehicle-treated mice (data not shown).

In response to inhaled metacholine in awake mice, BHR was also assessed by measuring the enhanced pause (PenH) using wholebody plethysmograpy. BHR to inhaled metacholine was similarly attenuated by iloprost treatment (Figure 1F).

Effect of iloprost inhalation prior to allergen challenge on DC function. As the above data suggested a strong antiinflammatory effect of iloprost, and as it has been previously reported that PGs can affect DC function (24), we addressed the question of whether local iloprost would modify DC function in the airways. Migration of DCs to the MLNs upon allergen encounter is a known function of DCs $(25,26)$. The total number of DCs in draining MLNs was enumerated 24 hours after the last OVA challenge. The number of DCs was greatly enhanced in OVA-sensitized mice subjected to OVA challenge compared with sham-sensitized mice (Figure 2A). Interestingly, iloprost treatment prior to each allergen challenge markedly decreased the number of DCs (MHCII ${ }^{\text {high }} \mathrm{CD} 11 \mathrm{c}^{\text {high }}$ ) in the draining LNs, and this was accompanied by a strong decrease in the number of $\mathrm{CD}^{+} \mathrm{CD}^{+}$and $\mathrm{CD}^{+} \mathrm{CD}^{+} \mathrm{T}$ cells and CD19+ $\mathrm{B}$ cells (Figure $2 \mathrm{~B}$ ).

In peripheral tissues such as the lung, DCs are found in a socalled immature state, expressing low levels of costimulatory molecules. Maturation of DCs is a key step for the induction and maintenance of allergic airway inflammation and has previ- ously been shown to occur locally in the airways of allergen-challenged mice $(22,27,28)$. Maturation is heralded by the expression of costimulatory molecules necessary for optimal $\mathrm{T}$ cell expansion and differentiation. As shown in Figure 2C, inhaled iloprost markedly reduced the expression of CD40, CD80, $\mathrm{CD} 86$, and CD83 on CD11 $\mathrm{c}^{+} \mathrm{MHCII}^{+}$lung DCs of allergen-challenged mice. In the MLNs, there were no significant differences in CD40, CD80, CD83, or CD86 expression between iloprostand vehicle-treated mice (data not shown). The effects of iloprost were most likely a direct effect on lung DC function, as in bone marrow-derived DCs exposed to OVA in vitro, iloprost significantly decreased the expression of CD40, CD80, CD83, and CD86 (Supplemental Figure 2A). Furthermore, the effect of iloprost on the expression of costimulatory molecules seemed to be DC specific, as treatment of mice with iloprost did not change the expression of CD40, CD80, or CD86 on lung macrophages, BAL macrophages, and BAL B cells (Supplemental Figure 2, $\mathrm{B}$ and $\mathrm{C}$, and data not shown).

Effect of iloprost inhalation on migration of lung DCs to the MLNs. A reduction in DC numbers in the MLNs of iloprost-treated mice could result from a reduction in lung inflammation and concomitant reduction in DC influx into the node (29). Alternatively, iloprost might also directly interfere with the migratory capacity of lung DCs in vivo. To more directly prove an effect of iloprost on the migration of lung DCs, fluorescently labeled OVA was injected 

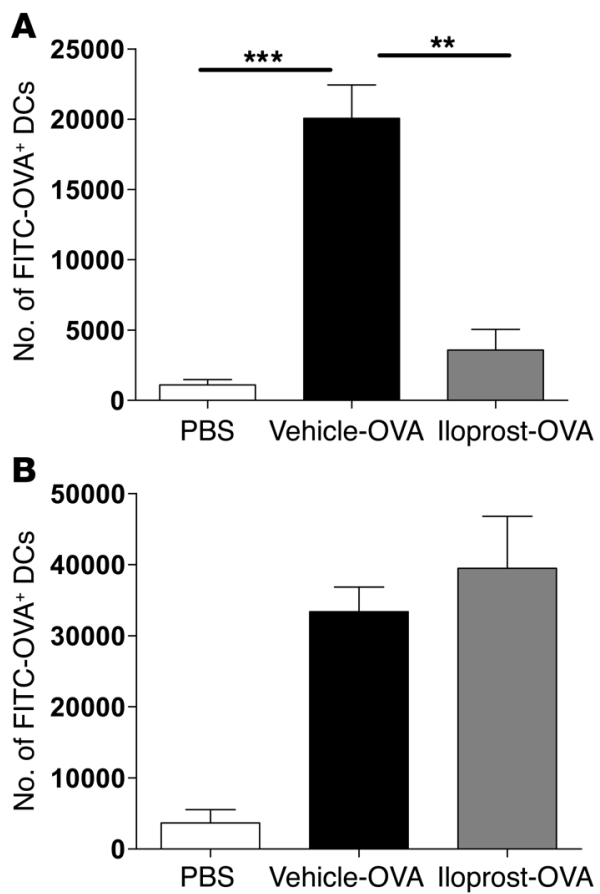

\section{Figure 3}

Effect of iloprost on lung DC migration to the thoracic draining LNs. (A) On day 0 , naive mice were instilled i.t. with FITC-OVA with or without $0.2 \mu \mathrm{g}$ iloprost. On day 1 , the presence of $\mathrm{FITC}^{+}$migrating DCs in thoracic draining LNs was analyzed by flow cytometry. (B) In the same mice, lung DCs were also enumerated. Lungs were enzymatically digested and stained for the presence of $\mathrm{FITC}+\mathrm{MHCll}+\mathrm{CD} 11 \mathrm{C}^{+}$ DCs. Results are representative of 4 mice per group. Experiments were repeated 3 times with similar results. Data are mean \pm SEM. ${ }^{* *} P<0.01 ;{ }^{* *} P<0.001$.

i.t. together with iloprost or vehicle alone in naive $\mathrm{BALB} / \mathrm{c}$ mice. As previously reported, the transport of the large molecule FITC-OVA is an exclusive function of lung-derived DCs, which transport this complex across the epithelial tight junction barrier; FITC-OVA starts to appear in the LNs 12 hours after injection $(19,30)$. The number of $\mathrm{MHCII}^{+} \mathrm{CD} 11 \mathrm{c}^{+}$DCs carrying fluorescent FITC-OVA cargo was enumerated in the MLNs 1 day after instillation, and, as shown in Figure 3A, concomitant treatment with $0.2 \mu \mathrm{g}$ iloprost significantly inhibited, but did not completely abolish, the migration of lung DCs to the LNs. Moreover, to exclude a possible toxic effect of iloprost on lung DCs, lungs were digested and stained for $\mathrm{MHCII}^{+} \mathrm{CD} 11 \mathrm{c}^{+} \mathrm{DCs}$. The absolute number of DCs detected in the lungs of animals that were instilled with FITC-OVA together with iloprost was identical compared with animals given FITC-OVA alone, illustrating that DCs were not killed (Figure 3B).

In vitro experiments revealed further that iloprost-treated DCs were unable to migrate in response to CC chemokine receptor 7 (CCR7) agonist CC chemokine ligand 19 (CCL19; Supplemental Figure 3 ), suggesting that a loss of chemotactic responsiveness to LN-expressed CCL19 was a likely cause for reduced migration of DCs to the draining LNs.

Effect of iloprost inhalation on the capacity of DCs to induce Th2 immune responses in the lung. The above data suggested that the inhibition of allergic airway inflammation by iloprost treatment during the allergen challenge phase was caused by alteration of lung DC func- tion, critical for mounting Th2 effector responses in sensitized mice (22). To prove more directly that local iloprost can suppress the function of lung DCs in vivo, we used a model in which Th2 sensitization depends on endogenous lung myeloid DCs ( $\mathrm{mDCs}$ ) (30). Mice were first depleted of tolerogenic plasmacytoid DCs (pDCs) by treatment with anti-Gr-1 Abs, thus leading to priming against inhaled harmless OVA. In Gr-1-treated mice, but not in control isotype-treated mice, OVA inhalation led to strong airway eosinophilia and lymphocytosis as well as goblet cell hyperplasia (Figure 4, A and B). This was accompanied by Th2 cytokine production in the MLNs (Figure 4C). Mice depleted of pDCs that were treated with $0.2 \mu \mathrm{g}$ iloprost at the time of OVA priming developed no signs of airway inflammation and had 5-fold reduced levels of cytokines in the LNs, thus suggesting that iloprost interferes with the potential of lung DCs to induce Th2 priming.

However, as iloprost was given via the airways, it would be possible that the inhibitory effects on DCs were indirect and were mediated by structural cells or effects on lymphocytes $(8,31-33)$. To rule out these indirect effects of iloprost treatment on lung structural cells such as epithelial cells or on lymphocytes, we performed experiments in which $\mathrm{mDCs}$ were treated in vitro with iloprost prior to adoptive transfer to the airways of naive mice. As the number of DCs obtained from the lungs of mice was too small to perform large adoptive transfer experiments, we used bone marrow-derived DCs grown in GM-CSF. As previously reported, adoptive i.t. transfer of these OVApulsed mDCs leads to Th2 priming and subsequent features of asthma upon OVA aerosol challenge 10 days later, a function that is related to the number of DCs injected $(18,34)$. In mice receiving unpulsed mDCs, few inflammatory cells accumulated in the BAL fluid and lung tissues after OVA aerosol challenge (Figure 5, A and B). In contrast, in mice receiving OVA-pulsed $\mathrm{mDCs}$, a strong cellular recruitment of lymphocytes and eosinophils occurred in the BAL fluid and peribronchial and perivascular area in the lung, accompanied by goblet cell hyperplasia. The pretreatment of OVA-pulsed mDCs with iloprost ex vivo significantly abolished the potential of these cells to induce eosinophilic airway inflammation and goblet cell hyperplasia (Figure 5B), and this was accompanied by a significant decrease in the level of IL-4, IL-5, and IL-13 in the MLNs, while the concentration of IFN- $\gamma$ was not significantly changed (data not shown). These data suggest that iloprost treatment of mDCs profoundly inhibits Th2 sensitization. To more directly address this point, we tested the effect of iloprost on DC-driven cytokine production of OVAspecific $\mathrm{T}$ cells in vivo (Figure 5C). In order for us to follow primary $\mathrm{T}$ cell activation, mice first received a cohort of naive OVA-TCR Tg (DO11.10) T cells, followed 2 days later by an i.t. injection of vehicletreated OVA-pulsed mDCs, iloprost-treated OVA-pulsed mDCs, or control unpulsed mDCs. DCs were extensively washed to avoid carryover of iloprost in vivo. LN cells (containing OVA-specific T cells) of mice immunized with iloprost-treated OVA-pulsed DCs produced lower levels of the Th2 cytokines IL-4, IL-5, and IL-13 than did cells stimulated with vehicle-treated OVA-pulsed DCs, while the production of IFN- $\gamma$ and IL-10 was increased. The same effects were seen when iloprost-treated OVA-pulsed mDCs were cocultured in vitro with purified naive (DO11.10) OVA-specific T cells, illustrating that the observed inhibition of Th2 development were inherent to DCs and not due to effects of iloprost on recruitment of $\mathrm{T}$ cells in vivo (Supplemental Figure 4).

Effect of in vitro iloprost treatment on the capacity of DCs to activate primed Th2 effector cells. In our initial experiments (Figure 1) we administered iloprost to the airways of already sensitized mice, 


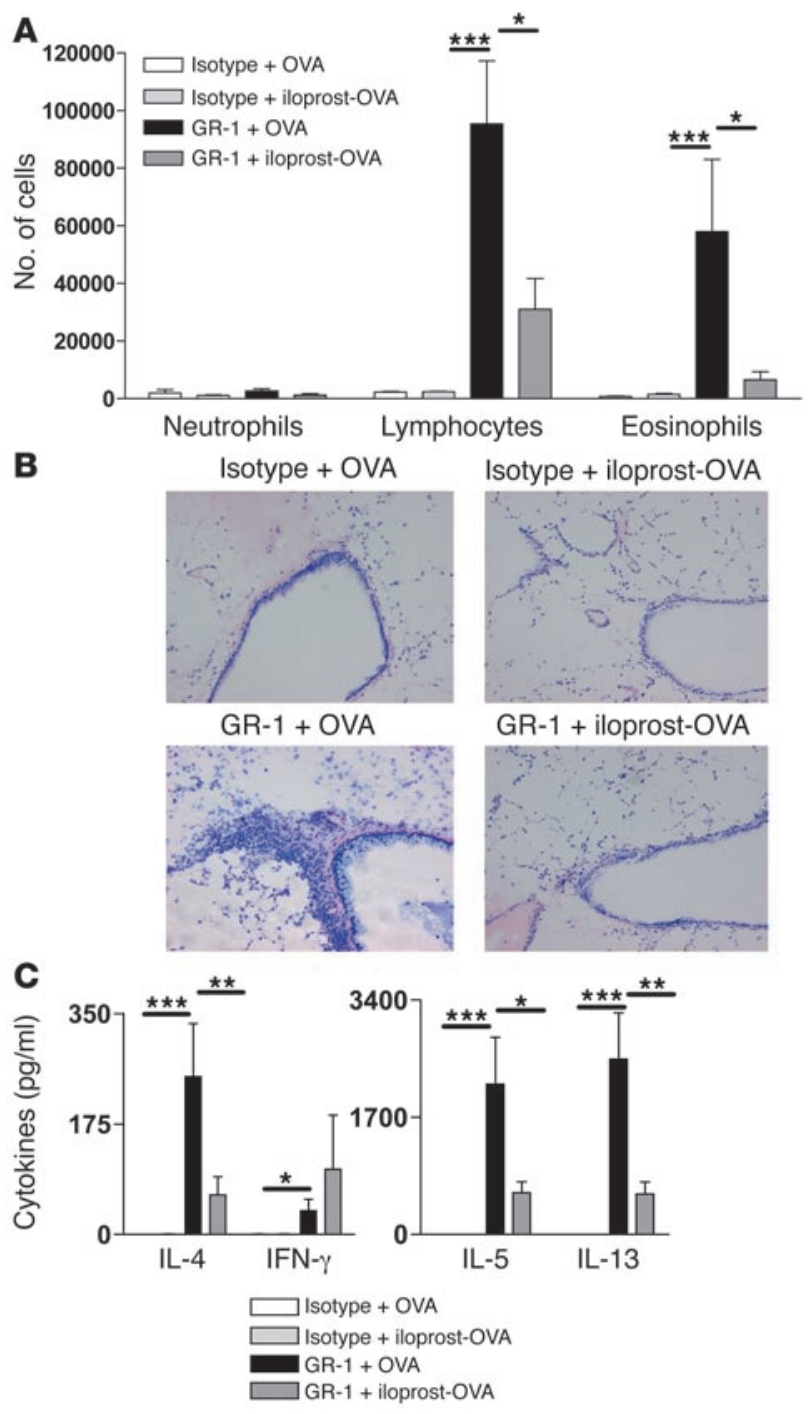

leading to a reduction of DC maturation (Figure 2C) and a consequent reduction in airway inflammation. However, these effects could also be due to effects of $\mathrm{PGI}_{2}$ on T cells, as it has previously been reported that the IP receptor is expressed on Th2 cells (8). To test whether iloprost-exposed DCs would be less efficient in stimulating effector Th2 cells, Th2 effector cells were made in vitro from cultures of DO11.10 OVA-specific T cells in the presence of OVA antigen and polarizing conditions (IL-4, anti-IFN- $\gamma$, and anti-IL-12) (22). When restimulated by OVA-pulsed DCs, these Th2 effector cells produced high amounts of IL-4, IL-5, and IL-13, but no IFN- $\gamma$ (Figure 6). However, treatment of OVA-pulsed DCs with $2.5 \mu \mathrm{g} / \mathrm{ml}$ iloprost prior to culture with Th2 cells strongly inhibited the production of Th2 cytokines.

\section{Discussion}

Apart from its dilatory effect on the pulmonary vessels and its antithrombotic effects, iloprost, a stable $\mathrm{PGI}_{2}$ analog, has been shown by several studies to have immune-regulatory effects by altering the production of cytokines and proinflammatory mediators and the expression of adhesion molecules (35-39). Iloprost and/or $\mathrm{PGI}_{2}$ suppress the function of various immune and inflammatory

\section{Figure 4}

Administration of iloprost prevents sensitization induced by DCs. On day 0 , mice received an i.t. injection of OVA in the presence or absence of iloprost. From days -1 to 2 , mice were injected i.p. with anti-Gr-1 Abs to deplete pDCs or isotype control Abs. Ten days later, mice were exposed to 3 OVA aerosols. (A) BAL fluid was analyzed by flow cytometry. (B) Hematoxylin and eosin staining of lung sections. (C) MLN cells restimulated in vitro for 4 days with OVA, and cytokines were measured in the supernatant. Data are mean \pm SEM. ${ }^{*} P<0.05$; ${ }^{* *} P<0.01 ;{ }^{* *} P<0.001$.

cells like monocytes/macrophages, lymphocytes, and neutrophils, which has led to the first clinical trials studying its effect as an antiinflammatory compound $(40,41)$. Orally administered iloprost can reduce the serum levels of TNF- $\alpha$ and soluble endothelial cell adhesion molecules (like VCAM-1 and ICAM-1) in vivo in patients with rheumatoid arthritis, and this is associated with improvement in the clinical course of the disease $(40,41)$.

In addition to oral administration, inhalation of aerosolized iloprost is a well-accepted and tolerated therapy for pulmonary arterial hypertension (15). The fact that iloprost can be given by inhalation would make it a feasible treatment option for asthmatic airway inflammation, as inhaled drugs such as corticosteroids and $\beta 2$-agonist bronchodilators constitute the standard therapy of asthma (42). Our present study demonstrates, for the first time to our knowledge, that inhaled iloprost via selective activation of IP receptors is indeed able to inhibit the salient features of experimental asthma, including Th2 cytokine production, eosinophilic airway inflammation, goblet cell hyperplasia, and BHR.

Several prior studies have suggested that $\mathrm{PGI}_{2}$ is released in the airways of allergen-challenged lungs and has an antiinflammatory effect on asthma $(3,8,11,12)$. The strongest evidence comes from studies in IP-deficient mice, which lack the sole receptor for $\mathrm{PGI}_{2}(13,14)$. In these mice, features of acute and chronic asthma (i.e., airway remodeling) were severely increased, but it was not reported how a defect in IP signaling led to an increase in inflammation. In addition, Jaffar et al. showed in a Th2 adoptive transfer model of asthma that a selective COX-2 inhibitor (NS-398) given at the time of allergen challenge led to a reduction in lung $\mathrm{PGI}_{2}$ levels and a consequent increased severity of asthmatic inflammation and BHR (8).

In all the studies reported so far, a clear mechanism by which endogenous $\mathrm{PGI}_{2}$ reduces inflammation has not been found. $\mathrm{PGI}_{2}$ has been shown to inhibit allergic mediator release and eosinophil recruitment in humans and experimental animals, but these effects could be indirect (43). Similarly, high levels of $\mathrm{PGI}_{2}$ are associated with less Th2 lymphocyte recruitment to the lungs, but again, these effects might be indirect due to a reduction in airway inflammation (8). Here we report that iloprost inhalation dramatically altered the function of antigen-presenting DCs. These cells are crucial for both the initiation and the maintenance phases of allergic asthma, as depletion of airway DCs during secondary challenge in sensitized mice abolished all cardinal features of asthma (including airway eosinophilia, goblet cell hyperplasia, and BHR to metacholine), an effect that could be completely restored by adoptive transfer of wild-type DCs (22). DCs are crucial for generating asthmatic inflammation because they can locally interact with Th2 effector cells in the airway wall by providing chemotactic cues for Th2 cells (CCL17 and CCL22) and by delivering MHC and costimulatory signals, 

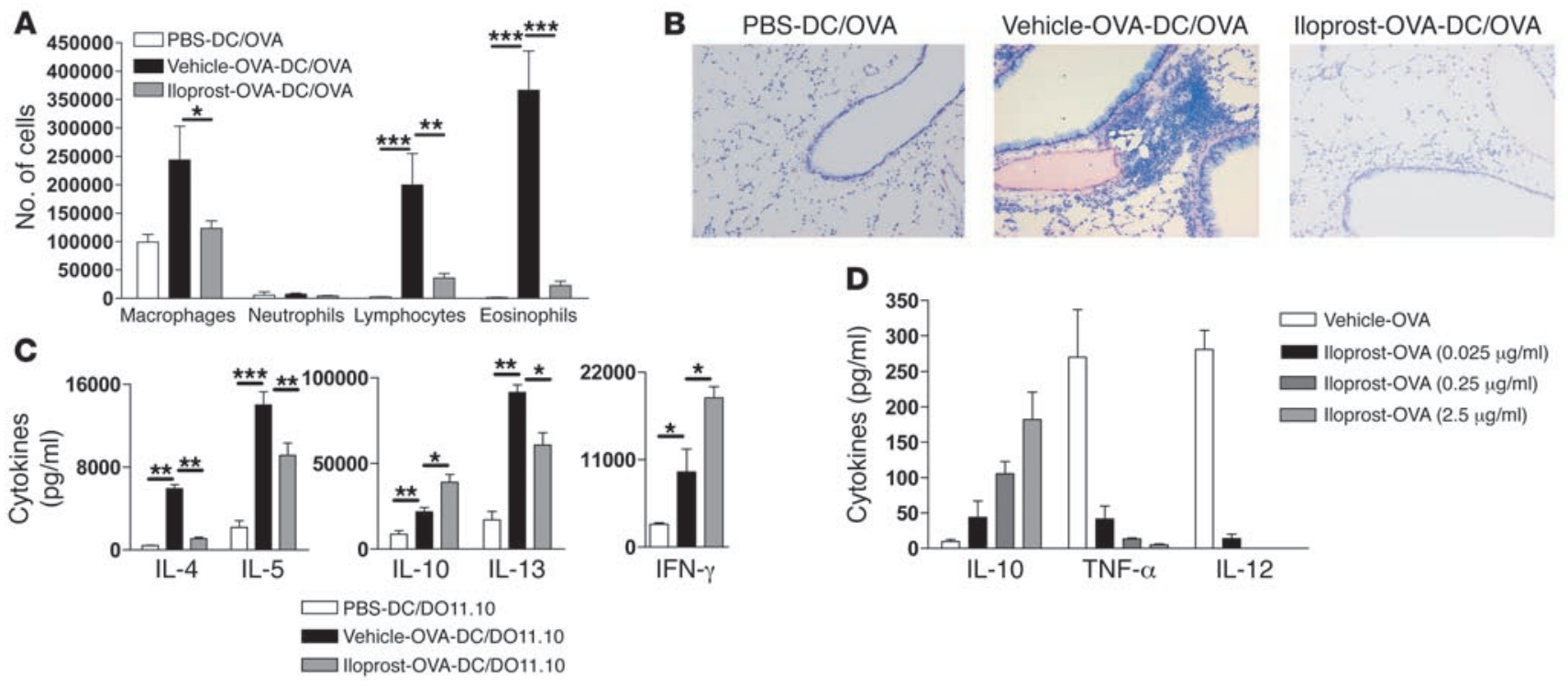

Figure 5

Iloprost treatment of DCs inhibits their potential to prime for Th2 responses. (A and B) On day 0 , mice received an i.t. injection of vehicle-treated OVA-pulsed DCs (vehicle-OVA-DC), iloprost-treated OVA-pulsed DCs, or unpulsed DCs. From days 10-13, all mice were exposed to OVA aerosols. (A) BAL fluid was analyzed by flow cytometry. (B) Hematoxylin and eosin staining of lung sections. (C) On day -2 , mice were injected i.v. with OVA-specific naive T cells from DO11.10 mice. On day 0, mice were instilled i.t. with vehicle-treated OVA-pulsed DCs, iloprost-treated OVA-pulsed DCs, or unpulsed DCs. On day 4, LN cells were collected and cultured in 96-well plates for 4 days. (D) Supernatants of bone marrow-derived DCs treated overnight with vehicle or different concentrations of iloprost were collected. The presence of IL-4, IL-5, IL-10, IL-12, TNF- $\alpha$, IL-13, and IFN- $\gamma$ in the supernatants was analyzed by ELISA. Data are mean \pm SEM. ${ }^{\star} P<0.05 ;{ }^{* \star} P<0.01 ;{ }^{* \star} P<0.001$.

thus triggering Th2 effector cytokine production $(27,44,45)$. Interestingly, iloprost treatment at the time of allergen challenge suppressed the expression of the costimulatory molecules CD40, CD80, CD86, and CD83, explaining how inflammation might be reduced. It was recently shown that CD80/CD86 costimulation on DCs is necessary for differentiation of Th2 cells from naive $\mathrm{T}$ cells and for restimulation of effector Th2 cells in the lung $(27,28,46)$. Although the precise role of CD83 in lung immunity is unknown, administration of soluble CD83 protein has previously been shown to completely inhibit DC-mediated T cell stimulation and severity of other T cell-mediated diseases (47). When we studied the effect of iloprost on bone marrow-derived DCs, we similarly observed a reduced expression of these costimulatory molecules, and these cells no longer induced Th2 differentiation or Th2 effector cytokine production in in vitro-differentiated Th2 cells (Figure 5C, Figure 6, and Supplemental Figure 4).

During allergen challenge in sensitized mice, DCs also migrate from the site of allergic inflammation to the draining LNs in order to induce expansion of recirculating central memory cells or stimulate naive T cells to become Th2 cells, thus feeding the inflammatory response with new waves of effector cells $(25,26,48)$. The treatment of mice with iloprost prior to allergen challenge suppressed this increase in MLN DCs. This could be due to a generalized reduction in the degree of airway inflammation in iloprosttreated mice, thus leading to reduced emigration from the lungs (29), but it could also be a direct effect of iloprost on the potential of lung DCs to migrate to these nodes. The latter concept is supported by our finding that iloprost also suppressed the migration of lung-derived FITC-OVA carrying DCs to the LNs in naive mice (Figure 3). It is well known that the migration of DCs from the periphery of the lung to the MLNs is CCR7 dependent $(49,50)$.
Iloprost treatment of DCs significantly reduced the chemotactic response toward the CCR7 ligand MIP3 $\beta$ in vitro, suggesting a direct effect of this compound on lung DC migration.

Although others suggested that $\mathrm{PGI}_{2}$ serves an antiasthmatic effect $(8,13,14)$, it is unclear at present whether $\mathrm{PGI}_{2}$ and IP signaling interfere with the sensitization phase of allergic asthma, as the IP-deficient mice had a constitutive deletion affecting both sensitization and challenge phases $(13,14)$ and the studies using COX-2 inhibitors employed a passive Th2 transfer model of asthma, in which the sensitization phase is bypassed (8). In allergic sensitization, when antigen is recognized for the first time by the cells of the pulmonary immune system, DCs play a crucial role. When properly triggered, $\mathrm{mDCs}$ promote $\mathrm{Th} 2$ priming while $\mathrm{pDCs}$ promote tolerance to inhaled antigen $(20,30,44)$. Inhalation of endotoxinlow OVA is a normally tolerogenic event in which pDCs inhibit the potential of mDCs to prime for effector Th2 cells $(30,44)$. By depleting pDCs, this tolerogenic response is turned into robust Th2 priming by $\mathrm{mDCs}$, and sensitization via the airways was observed. Under these conditions, iloprost completely abolished the development of Th2 effector cells; consequently, asthma did not develop upon repeated OVA challenge. The most likely explanation was a direct inhibitory effect of iloprost on lung DCs and not an indirect effect on epithelial cells. This notion is supported by the finding that the ex vivo pretreatment of OVA-pulsed mDCs prior to transfer to the airways significantly reduced their potential to induce Th2 priming. These effects were due to defective priming, as DO11.10 Th2 cell differentiation in the MLNs following i.t. injection of mDCs was severely impaired. Strikingly, however, the levels of the immunoregulatory cytokine IL-10 and the Th 1 cytokine IFN- $\gamma$ were increased. The same conclusions were reached when studying $\mathrm{T}$ cell differentiation in vitro. The effects of 


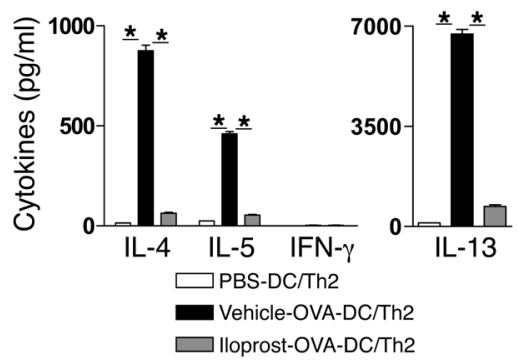

Figure 6

Iloprost-treated DCs fail to boost Th2 cytokine production in differentiated Th2 cells. Bone marrow-derived OVA-pulsed DCs were treated with lloprost or vehicle. DCs were collected and cocultured with in vitro differentiated D011.10 OVA-specific Th2 cells, that were previously generated in the presence of IL-4, anti-IL-12, and anti-IFN- $\gamma$. Cytokines were measured in the supernatant 4 days after setting up the culture. Data are mean \pm SEM. ${ }^{*} P<0.05$.

iloprost treatment on $\mathrm{T}$ cell differentiation could be due to reduced costimulatory molecule expression by DCs (Figure 2C), reduced migration of DCs to the LNs (Figure 3), or altered cytokine production following iloprost exposure. In bone marrow-derived DCs, iloprost indeed inhibited the production of IL-12 and TNF- $\alpha$, while it increased the release of IL-10 (Figure 5D), as previously shown (38). How this could affect Th2 priming is unclear at present, but clearly TNF- $\alpha$ might be required for optimal induction of a proinflammatory state (51).

In conclusion, the present study demonstrates for the first time to our knowledge that iloprost inhibits Th2-mediated cardinal features of asthma by altering the function of lung DCs. As iloprost inhalation is a well-tolerated and safe treatment for pulmonary hypertension, our findings of a therapeutic effect in experimental asthma should pave the way for a study addressing the effectiveness of this compound in humans with asthma. This paper validates the concept that targeting airway DC function is a powerful method to treat asthma.

\section{Methods}

Mice. BALB/c mice (6-8 weeks old) were purchased from Harlan. OVA-TCR $\mathrm{Tg}$ mice (DO11.10) on a BALB/c background were bred at the Erasmus Medical Center. All experimental protocols were approved by the animal ethics committee at the Erasmus Medical Center.

OVA/alum model of asthma. Mice were sensitized to OVA by i.p. injection of OVA/alum (10 $\mu$ g OVA grade $V$ adsorbed to $1 \mathrm{mg}$ aluminium hydroxide; Sigma-Aldrich) on days 0 and 7 and were challenged with OVA aerosols (grade III) on days 17-19, using a jet nebulizer delivering 1\% OVA in PBS for 30 minutes (21). Thirty minutes before each OVA exposure, mice were anesthetized with avertin and given an i.t. injection of control vehicle or $0.2 \mu \mathrm{g}$ iloprost (Cayman Biochemical Corp.). In some experiments mice were given an i.t. application of the selective IP receptor antagonist CAY10449 (200 $\mu \mathrm{g} / \mathrm{mouse})$ prior to iloprost or vehicle treatment.

Twenty-four hours after the last OVA exposure, BAL was performed 3 times, each time using $1 \mathrm{ml}$ of $\mathrm{Ca}^{+}$- and $\mathrm{Mg}^{+}$-free HBSS (Invitrogen) supplemented with $0.1 \mathrm{mM}$ sodium EDTA, followed by lung resection and storage in OCT freezing medium. In some experiments, lungs and MLNs were digested using collagenase/DNAse as described previously (19).

A mouse model of asthma induced by endogenous airway $m D C$. In order to study the effect of iloprost on Th2 sensitization induced by endogenous airway mDCs, mice were first depleted of tolerogenic pDCs by 3 i.p. injec- tions of the pDC-depleting Ab Gr-1 (RB6-8C5) on days -1, 0, and 1 as described previously (30). On day $0,800 \mu \mathrm{g}$ of OVA (LPS low; Worthington Biochemicals) was injected i.t., admixed with $2.5 \mu \mathrm{g}$ of iloprost or vehicle. After a washout period of 10 days, mice received 3 OVA aerosols of 30 minutes' duration on 3 consecutive days. Twenty-four hours later, BAL fluid, LNs, and lung tissues were taken.

A mouse model of asthma induced by adoptive transfer of bone marrow-derived $D C s$. We have previously reported on a model in which sensitization to inhaled OVA is induced by i.t. injection of OVA-pulsed bone marrowderived mDCs (18). DCs were prepared as previously described (18). Briefly, bone marrow cells were grown in RPMI 1640 supplemented with gentamycin, 2-mercaptoethanol, 5\% FCS (Biocell Laboratories), and recombinant murine GM-CSF (200 IU/ml). On days 3, 6, and 8, the medium was refreshed and GM-CSF was added. The purity of bone marrow-derived DCs was greater than $90 \%$.

On day 9 of culture, cells were pulsed overnight with $100 \mu \mathrm{g} / \mathrm{ml}$ LPS-low OVA (Worthington Biochemicals). Some plates were also treated with $2.5 \mu \mathrm{g} / \mathrm{ml}$ iloprost added 30 minutes before the addition of OVA. As a control, DCs were incubated with PBS containing the vehicle. After antigen pulsing, nonadherent DCs were collected, washed to remove free OVA or iloprost, and resuspended in PBS at a concentration of $12.5 \times 10^{6} \mathrm{cells} / \mathrm{ml}$.

For in vivo experiments, BALB/c mice were anesthetized on day 0 with avertin $\left(2 \% \mathrm{v} / \mathrm{v}\right.$ in PBS), and $1 \times 10^{6}$ vehicle-treated DCs, OVA-pulsed DCs, or iloprost-treated OVA-pulsed DCs were instilled through the opening vocal cords as described previously (18). On days 10-12, mice were exposed to a 30 -minute OVA aerosol. Mice were sacrificed 24 hours after the last aerosol.

Flow cytometry and sorting. After counting and washing, BAL cells were stained for 30 minutes with FITC-labeled anti-I-Ad/I-Ed (macrophages/ DCs), PE-labeled anti-CCR3 (eosinophils), Cy-chrome-labeled antiCD3 and anti-CD19 (lymphocytes), and allophycocyanin-labeled (APClabeled) anti-CD11c (macrophages/DCs) in PBS containing 0.5\% BSA and $0.01 \%$ sodium azide. Differential cell counts were analyzed by flow cytometry, as previously described (52).

For determination of lymphocytes and DC number in the MLNs, MLN cells were stained for $\mathrm{T}$ cell subsets (FITC-labeled anti-CD3, PE-labeled anti-CD4, Cy-chrome-labeled anti-CD8), B cells (PE-labeled anti-CD19) or DCs (FITC-labeled anti-MHCII, APC-labeled anti-CD11c). Absolute cell number was calculated by multiplying the total leukocyte number by the percentage of each population of interest.

For analysis of DC maturation, bone marrow, lung, or LN cell suspensions were stained with FITC-labeled anti-I-Ad/I-Ed; PE-labeled anti-CD40, anti-CD80, anti-CD83, and anti-CD86; and APC-labeled anti-CD11c Abs.

In order to address migration of lung DCs, $80 \mu \mathrm{l}$ of FITC-OVA $(10 \mathrm{mg} / \mathrm{ml})$, with or without iloprost, was administered i.t. (19). Control mice received $80 \mu \mathrm{l}$ of vehicle. At $24-36$ hours after injection, migrating DCs were enumerated in the MLNs as $\mathrm{CD} 11 \mathrm{c}^{+} \mathrm{MHCII}+$ cells carrying $\mathrm{FITC}^{+}$material.

In all experiments, dead cells were excluded from analysis using propidium iodide. Analysis was performed on a FacsCalibur flow cytometer (BD Biosciences) using Cellquest version 3.3 (BD Biosciences) and FlowJo version 6.4.7 (TreeStar Inc.) software.

Determination of BHR. Twenty-four hours after the last OVA aerosol challenge, nonspecific airway responsiveness was measured by exposing awake mice to aerosolized PBS to set a baseline value, followed by increasing concentrations of aerosolized metacholine (1.5625, 3.125, 6.25, 12.5, and 25 $\mathrm{mg} / \mathrm{ml}$ in PBS for 3 minutes; Sigma-Aldrich) using ultrasonic nebulizers. $\mathrm{PenH}$ values were measured for 3 minutes after each metacholine aerosol using a whole-body plethysmograph (Buxco Electronics). The average PenH values were expressed for each metacholine concentration as the percentage increase over baseline PenH values (53). 
For invasive measurement of dynamic resistance and compliance, mice were anesthetized with urethane, paralyzed using D-tubocurarine, tracheotomized, and intubated with an 18-gauge catheter, followed by mechanical ventilation with a Flexivent apparatus (SCIREQ). Respiratory frequency was set at 120 breaths per min with a tidal volume of $0.2 \mathrm{ml}$ and a positive end-expiratory pressure of $2 \mathrm{ml} \mathrm{H}_{2} \mathrm{O}$. Increasing concentrations of metacholine $\left(0-600 \mu \mathrm{g} / \mathrm{kg}^{-1}\right)$ were administered via the jugular vein. Dynamic resistance and compliance was recorded after a standardized inhalation maneuver given every 10 seconds for 2 minutes (54-56). Baseline resistance was restored before administering the subsequent doses of metacholine.

Effect of iloprost on the activation of OVA-specific naive $T$ cells in a primary immune response. Because the frequency of OVA-specific T cells is very low in naive animals, the primary activation of a naive $\mathrm{T}$ cell is difficult to detect. To avoid this problem, naive $\mathrm{T}$ cells purified from DO11.10 mice were adoptively transferred into BALB/c mice. Briefly, LNs and spleens were collected from DO11.10 mice and homogenized, and $10 \times 10^{6}$ live cells were injected i.v. in the lateral tail vein of $\mathrm{BALB} / \mathrm{c}$ mice (day -2 ). On day 0 , the mice received an i.t. injection of OVA-pulsed DCs, iloprost-treated OVA-pulsed DCs, or control unpulsed DCs. On day 4, MLNs were collected and homogenized, and LN cells (200,000 cells/well in triplicate) were resuspended in RPMI 1640 containing 5\% FCS and antibiotics and placed in 96-well plates. Four days later, supernatants were harvested and analyzed for the presence of IL-4, IL-5, IL-13, IL-10, and IFN- $\gamma$.

Activation of OVA-specific memory Th2 cells by mDCs. Naive CD4+ $\mathrm{T}$ cells $\left(1 \times 10^{5}\right)$ were purified from unmanipulated DO11.10 mice and were first differentiated for 7 days into effector Th2 cells in the presence of IL-4, anti-IFN- $\gamma$, and anti-IL-12, as previously described (22). After washing, these effector Th2 cells were cocultured with bone marrow- derived DCs $\left(1 \times 10^{4}\right)$, which were pretreated with iloprost or vehicle alone in round-bottomed 96-well tissue culture plates. After 4 days, supernatants were harvested and analyzed for the presence of IFN- $\gamma$, IL-4, IL-5, and IL-13 cytokines by ELISA.

Cytokine measurements. To measure cytokine levels, MLN cells were plated in round-bottomed 96 -well plates $\left(1 \times 10^{6} \mathrm{cells} / \mathrm{ml}\right)$ and restimulated with OVA $(10 \mu \mathrm{g} / \mathrm{ml})$ for 4 days. The presence of IL-4, IL-5, IL-13, IL-10, IL-12, TNF- $\alpha$, and IFN- $\gamma$ was assayed on supernatants by ELISA (BD).

Statistics. For all experiments, the difference between groups was calculated using the Mann-Whitney $U$ test for unpaired data. A $P$ value less than 0.05 was considered to be significant. Groups of mice consisted of at least 8 mice. Experiments were repeated at least 3 times.

\section{Acknowledgments}

This work was supported by Netherlands Organization of Scientific Research VIDI and VENI grants (to B.N. Lambrecht and H. Hammad, respectively) and by an Emmy Noether Fellowship to M. Idzko from the Deutsche Forschungsgemeinschaft (DFG ID7/3-1).

Received for publication April 28, 2006, and accepted in revised form December 5, 2006.

Address correspondence to: Bart N. Lambrecht, Department of Pulmonary Medicine, Room Ee2251a, Erasmus University Medical Center, Dr Molewaterplein 50, 3015 GE Rotterdam, The Netherlands. Phone: 31-10-408-7703; Fax: 31-10-408-9453; E-mail: b.lambrecht@erasmusmc.nl.

Marco Idzko and Hamida Hammad contributed equally to this work.
1. Park, G.Y., and Christman, J.W. 2006. Involvement of cyclooxygenase- 2 and prostaglandins in the molecular pathogenesis of inflammatory lung diseases. Am. J. Physiol. Lung Cell Mol. Physiol. 290:L797-L805.

2. Liu, M.C., et al. 1990. Evidence for elevated levels of histamine, prostaglandin D2, and other bronchoconstricting prostaglandins in the airways of subjects with mild asthma. Am. Rev. Respir. Dis. 142:126-132.

3. Dahlen, S.E., et al. 1983. Allergen challenge of lung tissue from asthmatics elicits bronchial contraction that correlates with the release of leukotrienes C4, D4, and E4. Proc. Natl. Acad. Sci. U. S. A. 80:1712-1716.

4. Carey, M.A., Germolec, D.R., Langenbach, R., and Zeldin, D.C. 2003. Cyclooxygenase enzymes in allergic inflammation and asthma. Prostaglandins Leukot. Essent. Fatty Acids. 69:157-162.

5. Warner, T.D., and Mitchell, J.A. 2004. Cyclooxygenases: new forms, new inhibitors, and lessons from the clinic. FASEB J. 18:790-804.

6. Helliwell, R.J., Adams, L.F., and Mitchell, M.D. 2004. Prostaglandin synthases: recent developments and a novel hypothesis. Prostaglandins Leukot. Essent. Fatty Acids. 70:101-113.

7. Kunikata, T., et al. 2005. Suppression of allergic inflammation by the prostaglandin $\mathrm{E}$ receptor subtype EP3. Nat. Immunol. 6:524-531.

8. Jaffar, Z., Wan, K.S., and Roberts, K. 2002. A key role for prostaglandin $\mathrm{I} 2$ in limiting lung mucosal Th2, but not Th1, responses to inhaled allergen. J. Immunol. 169:5997-6004.

9. Gavett, S.H., et al. 1999. Allergic lung responses are increased in prostaglandin $\mathrm{H}$ synthase-deficient mice. J. Clin. Invest. 104:721-732.

10. Peebles, R.S., Jr., et al. 2000. Cyclooxygenase inhibition increases interleukin 5 and interleukin 13 production and airway hyperresponsiveness in allergic mice. Am. J. Respir. Crit. Care Med. 162:676-681.

11. Walmrath, D., et al. 1991. Intravascular anti-IgE challenge in perfused lungs: mediator release and vascular pressor response. J. Appl. Physiol. 71:2499-2506.

12. Schulman, E.S., Newball, H.H., Demers, L.M., Fitzpatrick, F.A., and Adkinson, N.F., Jr. 1981. Anaphylactic release of thromboxane A2, prostaglandin D2, and prostacyclin from human lung parenchyma. Am. Rev. Respir. Dis. 124:402-406.

13. Takahashi, Y., et al. 2002. Augmentation of allergic inflammation in prostanoid IP receptor deficient mice. Br. J. Pharmacol. 137:315-322.

14. Nagao, K., et al. 2003. Role of prostaglandin I2 in airway remodeling induced by repeated allergen challenge in mice. Am. J. Respir. Cell Mol. Biol. 29:314-320.

15. Hoeper, M.M., et al. 2000. Long-term treatment of primary pulmonary hypertension with aerosolized iloprost, a prostacyclin analogue. N. Engl. J. Med. 342:1866-1870.

16. Banchereau, J., and Steinman, R.M. 1998. Dendritic cells and the control of immunity. Nature. 392:245-252

17. Lambrecht, B.N., and Hammad, H. 2003. Taking our breath away: dendritic cells in the pathogenesis of asthma. Nat. Rev. Immunol. 3:994-1003.

18. Lambrecht, B.N., et al. 2000. Myeloid dendritic cells induce Th 2 responses to inhaled antigen, leading to eosinophilic airway inflammation. J. Clin. Invest. 106:551-559.

19. Vermaelen, K.Y., Carro-Muino, I., Lambrecht, B.N., and Pauwels, R.A. 2001. Specific migratory dendritic cells rapidly transport antigen from the airways to the thoracic lymph nodes. J. Exp. Med. 193:51-60.

20. Eisenbarth, S.C., et al. 2002. Lipopolysaccharideenhanced, toll-like receptor 4-dependent T helper cell type 2 responses to inhaled antigen. J. Exp. Med. 196:1645-1651.
21. Lambrecht, B.N., Salomon, B., Klatzmann, D., and Pauwels, R.A. 1998. Dendritic cells are required for the development of chronic eosinophilic airway inflammation in response to inhaled antigen in sensitized mice. J. Immunol. 160:4090-4097.

22. van Rijt, L.S., et al. 2005. In vivo depletion of lung $\mathrm{CD} 11 \mathrm{c}+$ dendritic cells during allergen challenge abrogates the characteristic features of asthma. J. Exp. Med. 201:981-991.

23. Clark, R.D., et al. 2004. Discovery and SAR development of 2-(phenylamino) imidazolines as prostacyclin receptor antagonists [corrected]. Bioorg. Med. Chem. Lett. 14:1053-1056.

24. Morelli, A.E., and Thomson, A.W. 2003. Dendritic cells under the spell of prostaglandins. Trends Immunol. 24:108-111.

25. van Rijt, L.S., et al. 2002. Allergen-induced accumulation of airway dendritic cells is supported by an increase in CD31(hi)Ly-6C(neg) bone marrow precursors in a mouse model of asthma. Blood. 100:3663-3671.

26. Vermaelen, K., and Pauwels, R. 2003. Accelerated airway dendritic cell maturation, trafficking, and elimination in a mouse model of asthma. Am.J. Respir. Cell Mol. Biol. 29:405-409.

27. Huh, J.C., et al. 2003. Bidirectional interactions between antigen-bearing respiratory tract dendritic cells (DCs) and T cells precede the late phase reaction in experimental asthma: DC activation occurs in the airway mucosa but not in the lung parenchyma. J. Exp. Med. 198:19-30.

28. Lewkowich, I.P., et al. 2005. CD4+CD25+ T cells protect against experimentally induced asthma and alter pulmonary dendritic cell phenotype and function. J. Exp. Med. 202:1549-1561.

29. Martin-Fontecha, A., et al. 2003. Regulation of dendritic cell migration to the draining lymph node: impact on T lymphocyte traffic and priming. J. Exp. Med. 198:615-621. 
30. de Heer, H.J., et al. 2004. Essential role of lung plasmacytoid dendritic cells in preventing asthmatic reactions to harmless inhaled antigen. J. Exp. Med. 200:89-98.

31. Marcinkiewicz, J., and Chain, B.M. 1993. Differential cytokine regulation by eicosanoids in T cells primed by contact sensitisation with TNP. Cell. Immunol. 149:303-314.

32. Della Bella, S., et al. 1997. Differential effects of cyclo-oxygenase pathway metabolites on cytokine production by T lymphocytes. Prostaglandins Leukot. Essent. Fatty Acids. 56:177-184.

33. Costantini, V., et al. 1990. Effects of a stable prostacyclin analogue on platelet activity and on host immunocompetence in mice. Prostaglandins. 39:581-599.

34. Depuydt, P.O., Lambrecht, B.N., Joos, G.F., and Pauwels, R.A. 2002. Effect of ozone exposure on allergic sensitization and airway inflammation induced by dendritic cells. Clin. Exp. Allergy. 32:391-396.

35. Czeslick, E.G., Simm, A., Grond, S., Silber, R.E., and Sablotzki, A. 2003. Inhibition of intracellular tumour necrosis factor (TNF)-alpha and interleukin (IL)-6 production in human monocytes by iloprost. Eur. J. Clin. Invest. 33:1013-1017.

36. Muzaffar, S., Shukla, N., Lobo, C., Angelini, G.D., and Jeremy, J.Y. 2004. Iloprost inhibits superoxide formation and gp91phox expression induced by the thromboxane A2 analogue U46619, 8-isoprostane F2alpha, prostaglandin F2alpha, cytokines and endotoxin in the pig pulmonary artery. Br. J. Pharmacol. 141:488-496.

37. Mazzone, A., et al. 2002. Effects of iloprost on adhesion molecules and $\mathrm{F} 1+2$ in peripheral ischemia. Eur. J. Clin. Invest. 32:882-888.

38. Jozefowski, S., Bobek, M., and Marcinkiewicz, J. 2003. Exogenous but not endogenous prostanoids regulate cytokine secretion from murine bone mar- row dendritic cells: EP2, DP, and IP but not EP1, EP3, and FP prostanoid receptors are involved. Int Immunopharmacol. 3:865-878.

39. Rose, F., et al. 2003. Increased neutrophil mediator release in patients with pulmonary hypertension suppression by inhaled iloprost. Thromb. Haemost. 90:1141-1149.

40. Boehme, M.W., Gao, I.K., Norden, C., and Lemmel, E.M. 2006. Decrease in circulating endothelia cell adhesion molecule and thrombomodulin levels during oral iloprost treatment in rheumatoid arthritis patients: preliminary results. Rheumatol. Int. 26:340-347.

41. Gao, I.K., Scholz, P., Boehme, M.W., Norden, C., and Lemmel, E.M. 2002. A 7-day oral treatment of patients with active rheumatoid arthritis using the prostacyclin analog iloprost: cytokine modulation, safety, and clinical effects. Rheumatol. Int. 22:45-51.

42. Barnes, P.J. 2004. New drugs for asthma. Nat. Rev. Drug Discov. 3:831-844.

43. Lellouch-Tubiana, A., Lefort, J., Simon, M.T., Pfister, A., and Vargaftig, B.B. 1988. Eosinophil recruitment into guinea pig lungs after PAF-acether and allergen administration. Modulation by prostacyclin, platelet depletion, and selective antagonists. Am. Rev. Respir. Dis. 137:948-954.

44. Kohl, J., et al. 2006. A regulatory role for the C5a anaphylatoxin in type 2 immunity in asthma. J. Clin. Invest. 116:783-796. doi:10.1172/JCI26582.

45. Zhou, B., et al. 2005. Thymic stromal lymphopoietin as a key initiator of allergic airway inflammation in mice. Nat. Immunol. 6:1047-1053.

46. van Rijt, L.S., et al. 2004. Essential role of dendritic cell CD80/CD86 costimulation in the induction, but not reactivation, of $\mathrm{TH} 2$ effector responses in a mouse model of asthma. J. Allergy Clin. Immunol. 114:166-173.

47. Zinser, E., Lechmann, M., Golka, A., Lutz, M.B., and Steinkasserer, A. 2004. Prevention and treatment of experimental autoimmune encephalomyelitis by soluble CD83. J. Exp. Med. 200:345-351.

48. Harris, N.L., Watt, V., Ronchese, F., and Le Gros, G. 2002. Differential T cell function and fate in lymph node and nonlymphoid tissues. J. Exp. Med. 195:317-326.

49. Hammad, H., et al. 2002. Monocyte-derived dendritic cells induce a house dust mite-specific Th2 allergic inflammation in the lung of humanized SCID mice: involvement of CCR7. J. Immunol. 169:1524-1534

50. Sanchez-Sanchez, N., Riol-Blanco, L., and RodriguezFernandez, J.L. 2006. The multiple personalities of the chemokine receptor CCR7 in dendritic cells. J. Immunol. 176:5153-5159.

51. Serbina, N.V., Salazar-Mather, T.P., Biron, C.A., Kuziel, W.A., and Pamer, E.G. 2003. TNF/iNOSproducing dendritic cells mediate innate immune defense against bacterial infection. Immunity. 19:59-70.

52. van Rijt, L.S., et al. 2004. A rapid flow cytometric method for determining the cellular composition of bronchoalveolar lavage fluid cells in mouse models of asthma. J. Immunol. Methods. 288:111-121.

53. Hamelmann, E., et al. 1997. Noninvasive measurement of airway responsiveness in allergic mice using barometric plethysmography. Am. J. Respir. Crit. Care Med. 156:766-775.

54. Hantos, Z., Daroczy, B., Suki, B., Nagy, S., and Fredberg, J.J. 1992. Input impedance and peripheral inhomogeneity of dog lungs. J. Appl. Physiol. 72:168-178.

55. Fredberg, J.J., and Stamenovic, D. 1989. On the imperfect elasticity of lung tissue. J. Appl. Physiol. 67:2408-2419.

56. Collins, R.A., et al. 2005. Hyperresponsiveness to inhaled but not intravenous methacholine during acute respiratory syncytial virus infection in mice. Respir. Res. 6:142. 